\title{
Recent Patents on Nanosensor for Tumor Biomarker Detection
}

\author{
Weijun Shao ${ }^{1}$, Shu Zheng ${ }^{2, *}$, Lifeng Qi ${ }^{1, *}$ \\ ${ }^{1}$ Zhejiang California Nanosystems Institute, Zhejiang University. No. 268, Kaixuan Road, Hangzhou, Zhejiang, China, 310029. \\ ${ }^{2}$ The 2nd Affiliated Hospital, School of Medicine, College of Life Sciences,Zhejiang University, No. 88, Jiefang Road, Hangzhou, China \\ * Corresponding authors: qlfzju@gmail.com (Lifeng Qi) and zhengshu@zju.edu.cn (Shu Zheng)
}

\begin{abstract}
Since cancer has been the biggest threat to our human beings' health, more and more new patents of medical treatments for cancer detection have been used to make us out of suffering from cancer. Currently, most of patients check out their cancer cell at the late stage when cure is difficult, so early detection of cancer has been demonstrated to significantly important for improving clinical outcomes. But the current clinical methods available for such assessments are slow, expensive and inaccurate. There is a new development of nanosensor technology which makes it possible to detect the tumor earlier. The present paper summarizes recent new discoveries of nanosenor for tumor biomarker detection which allow for low-cost, minimally invasive approach with high sensitivity and specificity by detecting tumor biomarkers from the exhaled breath, proteomic, and molecule etc.
\end{abstract}

Keywords: Nanosensor, Tumor Biomarker, Detection

Citation: W. Shao, et al. Recent Patents on Nanosensor for Tumor Biomarker Detection. Nano Biomed. Eng. 2011, 3(1), 66-72. DOI: 10.5101/nbe.v3i1.p66-72.

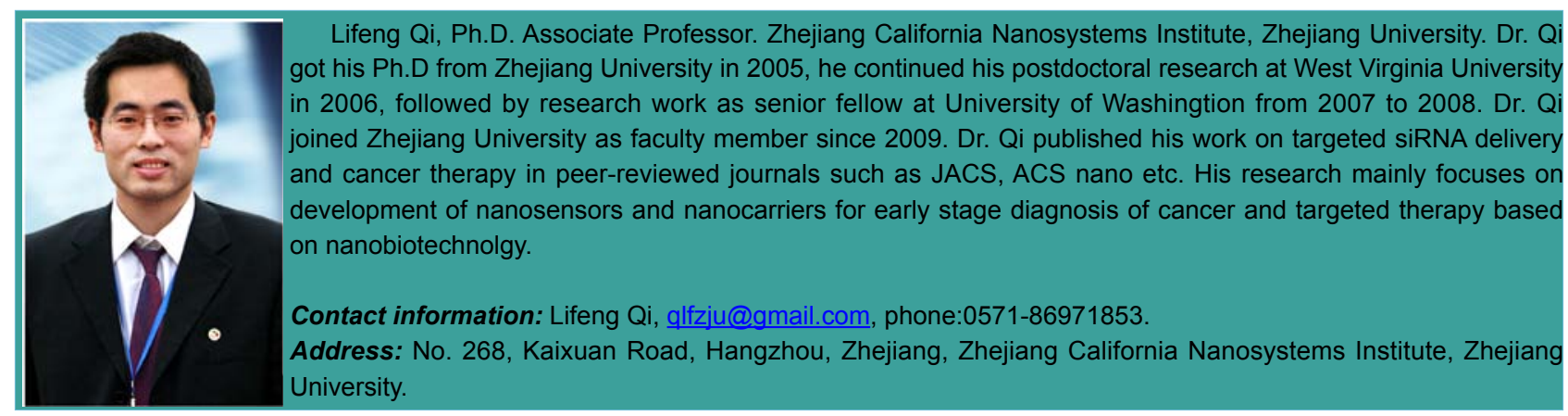

\section{Introduction}

Cancer is one of the most lethal diseases. This largely reflects the fact that approximately $75 \%$ of cases are detected at advanced stages of disease, when cure is unlikely. In contrast, 5 -year survival for patients with early stage cancers, such as ovarian cancer, breast cancer and lung cancer etc., can exceed $90 \%$. It is possible therefore that detecting a greater number of patients with early stage disease by novel and advanced tumor detection technologies which could significantly improve overall survival.

Early detection of cancer has been demonstrated to significantly improve clinical outcomes. Advances in cancer biology and technology support are increasingly important, recently people use many methods to detect the tumor cells, such as nano imaging, proteomic pattern analysis, nanosensor, gene chip sensor, SPR, etc. among the above mentioned methods, Nanotechnology, in particular nanosensor, offers many advantages when used for applications such as the delivery of bioactive agents (i.e., DNA, AIDS drugs, gene therapy, immunosuppressants, chemotherapeutics), drug uptake and degradation (Le., enzyme encapsulation), and tumor biomarker detection. This review summarizes the recent patents listed in Table 1 on the development of nanosenser for tumor biomarker detection.

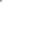


Table 1. Patents on Nanosensor for Tumor Biomarker Detection.

\begin{tabular}{|c|c|c|c|c|c|c|}
\hline & Patent Number & Description/Title & Assignee & Inventor & $\begin{array}{l}\text { Priority } \\
\text { Application } \\
\text { Date }\end{array}$ & $\begin{array}{l}\text { Publication } \\
\text { Date }\end{array}$ \\
\hline 1 & WO2009144725 & $\begin{array}{c}\text { CARBON NANOTUBE } \\
\text { STRUCTURES IN SENSOR } \\
\text { APPARATUSES FOR ANALYZING } \\
\text { BIOMARKERS IN BREATH } \\
\text { SAMPLES }\end{array}$ & $\begin{array}{c}\text { WEBB \& ASSOCIATES; P.O. Box } \\
218976121 \text { Rehovot (IL) }\end{array}$ & $\begin{array}{l}\text { HAICK, Hossam; (IL). } \\
\text { GANG, Peng; (IL). }\end{array}$ & $29 / 05 / 2008$ & 03/12/2009 \\
\hline 2 & WO2007086986 & $\begin{array}{c}\text { NOVEL APPLICATION OF } \\
\text { BIOSENSORS FOR DIAGNOSIS AND } \\
\text { TREATMENT OF DISEASE }\end{array}$ & \begin{tabular}{|} 
SALIWANCHIK, David, R. et al.; \\
Saliwanchik, Lloyd \& Saliwanchik, \\
A Professional Association, P.O. \\
Box 142950, Gainesville, FL 32614- \\
2950 (US).
\end{tabular} & $\begin{array}{l}\text { MELKER, Richard, J.; (US). } \\
\text { DENNIS, Donn, Michael; (US). }\end{array}$ & $07 / 12 / 2005$ & $02 / 08 / 2007$ \\
\hline 3 & WO2009053981 & \begin{tabular}{|c|} 
ELECTRONIC NOSE DEVICE \\
WITH SENSORS COMPOSED OF \\
NANOWIRES OF COLUMNAR \\
DISCOTIC LIQUID CRYSTALS WITH \\
LOW SENSITIVITY TO HUMIDITY
\end{tabular} & $\begin{array}{c}\text { WEBB \& ASSOCIATES et al.; P.O. } \\
\text { Box 2189, } 76121 \text { Rehovot (IL) }\end{array}$ & HAICK, Hossam; (IL). & $23 / 10 / 2007$ & $30 / 04 / 2009$ \\
\hline 4 & WO2008124525 & $\begin{array}{l}\text { NANOPARTICLES WITH } \\
\text { MOLECULAR RECOGNITION } \\
\text { ELEMENTS }\end{array}$ & \begin{tabular}{|c|} 
MYERS JR., Richard S. et al.; Stites \\
\& Harbison PLLC, 401 Commerce \\
Street, Suite 800, Nashville, \\
Tennessee 37219 (US).
\end{tabular} & $\begin{array}{l}\text { SOMAN, Chinmay Prakash; (US). } \\
\text { GIORGIO, Todd Donald; (US). }\end{array}$ & $\begin{array}{l}" 03 / 04 / 2007 \\
15 / 02 / 2008 "\end{array}$ & $16 / 10 / 2008$ \\
\hline 5 & WO2009123934 & $\begin{array}{c}\text { BRANCHED MULTIFUNCTIONAL } \\
\text { NANOPARTICLE CONJUGATES } \\
\text { AND THEIR USE }\end{array}$ & NOONAN, William, D.; (US) . & $\begin{array}{l}\text { NIE, Shuming; (US). } \\
\text { DUAN, Hongwei; (US). } \\
\text { KUANG, Min; (US). }\end{array}$ & $29 / 03 / 2008$ & $08 / 10 / 2009$ \\
\hline 6 & WO2009013754 & $\begin{array}{c}\text { CHEMICALLY SENSITIVE FIELD } \\
\text { EFFECT TRANSISTORS AND USE } \\
\text { THEREOF IN ELECTRONIC NOSE } \\
\text { DEVICES }\end{array}$ & $\begin{array}{l}\text { WEBB \& ASSOCIATES; P.O. Box } \\
2189,76121 \text { Rehovot (IL). }\end{array}$ & HAICK, Hossam; (IL). & $\begin{array}{l}\text { "24/07/2007 } \\
27 / 05 / 2008 "\end{array}$ & $29 / 01 / 2009$ \\
\hline 7 & WO2007094805 & $\begin{array}{c}\text { nanowire METHOD FOR } \\
\text { ELECTROCATALYTIC PROTEIN } \\
\text { DETECTION }\end{array}$ & $\begin{array}{c}\text { ANDERSON, MaryDilys; WOLF, } \\
\text { GREENFIELD \& SACKS, P.C., } 600 \\
\text { Atlantic Avenue, Boston, MA } 02210 \\
\text { (US) }\end{array}$ & $\begin{array}{l}\text { KELLEY, Shana, O.; (US). } \\
\text { ROBERTS, Marcel, A.; (US). }\end{array}$ & $12 / 04 / 2005$ & $23 / 08 / 2007$ \\
\hline 8 & WO2008018933 & $\begin{array}{c}\text { DETECTION OF PROTEASE AND } \\
\text { PROTEASE ACTIVITY USING A } \\
\text { SINGLE NANOCRESCENT SERS } \\
\text { PROBE }\end{array}$ & $\begin{array}{c}\text { HUNTER, Tom et al.; Beyer Weaver } \\
\text { LLP, P. O. Box 70250, Oakland, CA } \\
94612-0250 \text { (US) . }\end{array}$ & $\begin{array}{l}\text { LIU, Gang, L.; (US). } \\
\text { ELLMAN, Jonathan, A.; (US). } \\
\text { LEE, Luke, P.; (US). } \\
\text { CHEN, Fanqing, Frank; (US). }\end{array}$ & $03 / 05 / 2006$ & $14 / 02 / 2008$ \\
\hline 9 & WO2008140624 & $\begin{array}{l}\text { METHODS AND COMPOSITIONS } \\
\text { RELATED TO HYBIRD } \\
\text { NANOPARTICLES }\end{array}$ & $\begin{array}{c}\text { LANDRUM, Charles, P.; Fulbright } \\
\text { \& Jaworski L.L.P., 600 Congress } \\
\text { Avenue, Suite 2400, Austin, TX } \\
78701 \text { (US). }\end{array}$ & $\begin{array}{l}\text { SOKOLOV, Konstantin; (US). } \\
\text { MILNER, Thomas; (US). } \\
\text { LARSON, Timothy; (US). } \\
\text { BANKSON, James; (US). } \\
\text { AARON, Jesse; (US). } \\
\text { OH, Jungwan; . } \\
\text { JI, Xiaojun; . } \\
\text { LI, Chun; (US). }\end{array}$ & $22 / 12 / 2006$ & $20 / 11 / 2008$ \\
\hline 10 & WO2008122035 & $\begin{array}{l}\text { IN VIVO TUMOR TARGETING AND } \\
\text { SPECTROSCOPIC DETECTION } \\
\text { WITH SURFACE-ENHANCED } \\
\text { RAMAN NANOPARTICLE TAGS }\end{array}$ & $\begin{array}{l}\text { LINDER, Christopher, B.; Thomas, } \\
\text { Kayden, Horstemeyer \& Risley, } \\
\text { LLP, } 600 \text { Galleria Parkway, Suite } \\
\text { 1500, Atlanta, GA } 30339 \text { (US) . }\end{array}$ & $\begin{array}{l}\text { QUIAN, Ximei; . } \\
\text { ANSARI, Dominic; . } \\
\text { NIE, Shuming; (US). }\end{array}$ & $02 / 04 / 2007$ & 09/10/2008 \\
\hline 11 & WO2009086561 & NANO-GETTER DEVICE & $\begin{array}{c}\text { SCHWARZ, Paul, A.; Duane Morris } \\
\text { LLP, PO Box 5203, Princeton, NJ } \\
08543-5203 \text { (US) }\end{array}$ & $\begin{array}{l}\text { PALENA,Patricia,D.;(US). } \\
\text { BARTO,Richard,R.,Jr.;(US). } \\
\text { BORDERS,Tammie,L.;(US). } \\
\text { STUART,Jeffrey,A.;(US) }\end{array}$ & $03 / 01 / 2008$ & 09/07/2009 \\
\hline 12 & US20080038830 & $\begin{array}{l}\text { NANO-PARTICLE BIOCHIP } \\
\text { SUBSTRATES }\end{array}$ & $\begin{array}{l}\text { WOLF, GREENFIELD \& SACKS, } \\
\text { P.C., 600 Atlantic Avenue, Boston, } \\
\text { MA 02210-2206 (US) }\end{array}$ & $\begin{array}{l}\text { Ure, David A. (Somerville, MA, US). Palmer, } \\
\text { Richard E. (Hagley, GB). }\end{array}$ & 03/28/2007 & $02 / 14 / 2008$ \\
\hline 13 & US20060003465 & $\begin{array}{c}\text { LUMINESCENCE } \\
\text { ChARACTERIZATION OF } \\
\text { QUANTUM DOTS CONJUGATED } \\
\text { WITH BIOMARKERS FOR EARLY } \\
\text { CANCER DETECTION }\end{array}$ & $\begin{array}{l}\text { UNIVERSITY OF SOUTH } \\
\text { FLORIDA ( } 4202 \text { East Fowler } \\
\text { Avenue, Tampa, FL, US) }\end{array}$ & $\begin{array}{l}\text { Zhukov, Tatyana A. (Lutz, FL, US) } \\
\text { Ostapenko, Sergei (Wesley Chapel, FL, US) } \\
\text { Sutphen, Rebecca (Tampa, FL, US) } \\
\text { Lancaster, Johnathan (Tampa, FL, US) } \\
\text { Sellers, Thomas A. (Tampa, FL, US) } \\
\text { Zhang, Jin Z. (Santa Cruz, CA, US) }\end{array}$ & $06 / 30 / 2005$ & 01/05/2006 \\
\hline
\end{tabular}




\section{NANOSENSOR FOR TUMOR BIOMARKER IN BREATH SAMPLES}

\section{Using Nanotube Biosensor to Get Tumor Biomarkers in Breath Samples}

Breath testing has long been recognized as a reliable technique for diagnosing certain medical conditions through the detection of specific volatile metabolites in exhaled breath [1]. The analysis of breath offers several advantages being a non-invasive technique that possess the potential for direct and real-time monitoring [2].

Some inventors provide a system comprising an array of sensors for measuring volatile organic compounds as biomarkers for diagnostic and prognostic purposes, particularly, this system comprises an array of sensors comprising a (semi-) conductive random network of single-walled carbon nanotubes (SWCNTs) coated with small organic molecules in conjunction with learning and pattern recognition algorithms. This invention further provides methods of use thereof in measuring breath analytes indicative of cancer, e.g., lung cancer [3].

In another invention, the authors describe a unique method for detecting analytes/biomarkers of interest in exhaled breath by use of generally nanotubes. According to the invention, nanotubes containing volatile or "surrogate" biomarkers are provided that discharge the volatile or "surrogate" biomarkers from the nanotube under all kinds of conditions to diagnose and/or treat a disease. The invention makes use of molecular recognition agents in combination with a signaling agent (such as a molecular beacon, fluorescent polymer, or volatile biomarker) to non-invasively detect drugs, biomarkers, and other analytes in extremely low concentrations in exhaled breath and other bodily fluids. In a preferred embodiment, the molecular recognition agent is an aptamer, a highly specific nucleic acid-based ligand, that highly specific for a target analyte/biomarker. Prostate cancers generate a protein, prostate specific antigen (PSA). An aptamer could be designed that is specific for PSA (PSA-aptamer). The PSA-aptamer can be adhered to an end cap that fits on the end of a nanotube. A rapid test for the presence of prostate cancer, or a recurrence, could be developed where the volatile or "surrogate" biomarker is released from the nanotube after PSA (the biomarker of interest) interacts with the PSA-aptamer and "uncaps" the nanotubes. Using any of previously disclosed detector technologies, the volatile biomarker is detected in exhaled breath, which indicates the presence of PSA in the blood [4].

\section{Using Electronic Nose Device to Get Tumor Biomarkers in Breath Samples}

The following two inventions describe strategy that testing the volatile organic in normal human breath by using of electronic nose device. It is said when diseases such as lung cancer, liver disease, inflammatory bowel disease, and rheumatoid arthritis appear, an increase in 68 the level of oxidative stress markers can be observed [5, $6]$.

One invention relates to an electronic device comprises sensor of discotic liquid crystals in nanowire configuration and has low susceptibility towards humidity, and is thus more sensitive than known systems serving a similar purpose. The invention further provides a system comprising an electronic device comprising an array of chemically sensitive sensors of columnar discotic liquid crystal nanowires in conjunction with pattern recognition analyzer, wherein the pattern recognition analyzer uses methods such as artificial neural networks and principal component analysis to detect as well as quantify specific volatile organic compounds, without a need for sample pre-processing steps.

The invention is based in part on the unexpected finding that wires of columnar discotic liquid crystals can be used as sensors with improved sensing capabilities and essentially no sensitivity to humidity. The low sensitivity to humidity is achieved through the use of columnar discotic liquid crystals having a nanowire configuration wherein the surface of the nano wires is composed of hydrocarbons. In this unique configuration, elements that might be sensitive to humidity (e.g. oxygen, nitrogen), if present, are all confined to the core of the selfassembled nano wires. The device disclosed herein enable the detection of minute quantities of volatile organic compounds as biomarkers for diagnostic and prognostic purposes without the need for pre-concentrating or dehumidifying the sample [7].

\section{Chemically Sensitive Filed Effect Transistors in Eltectronic Nose Devices}

The other provides chemically sensitive field effect transistors of non-oxidized, functionalized silicon nanowires (Si, NWs) for detecting VOC (volatile organic compounds) with high sensitivity. In particular, the present invention further relates to a system comprising an electronic device comprising an array of chemically sensitive sensors in conjunction with learning and pattern recognition algorithms. The learning and pattern recognition algorithms receive sensor output signals which are analyzed using methods such as artificial neural networks and principal component analysis and are subsequently compared to stored data.

In a certain embodiment, the electronic devices disclosed herein detect volatile organic compounds with sensitivity of one part per billion (ppb) or less. In currently preferred embodiments the surface of the Si NWs is modified with a plurality of compounds via a direct $\mathrm{Si}-\mathrm{C}$ bond,these compounds form film layers on the surface of the nanowires, without intervening oxide layers. For example, the present invention uses compounds that attach throughSi-C-C, Si-C $=\mathrm{C}$, and $\mathrm{Si}-$ $\mathrm{C} \equiv \mathrm{C}$ bonds [8].

In a particular embodiment of these inventions, the Nano Biomed. Eng. 2011, 3(1),66-72 
method described herein is used to diagnose cancer. GasChromatography linked with Mass- Spectrometry (GCMS) studies have shown that volatile C4-C20 alkanes and certain monomethylated alkanes as well as benzene derivatives appear to be elevated in various instances of cancer. The compounds of interest are generally found in the range of $1-20 \mathrm{ppb}$ in healthy human breath, but can be seen in distinctive mixture compositions at elevated levels in the range of 10-100 ppb in the breath of diseased patients. The VOC levels are elevated even at the early stages of the disease, since they reflect a change in human body chemistry. This change appears regardless of the cancerous tumor size. In addition, biomarkers of a specific disease (e.g., lung cancer) possess distinctive mixture compositions/patterns in comparison to biomarkers of other diseases even those of closely related diseases (e.g., breast cancer).

\section{NANOPARTICLE AND NANOWIRE FOR PROTEOMIC BIOMARKER IN CANCER CELL}

This section provides us a method of discovering the proteomic biomarker in cancer cell by use of nanoparticles with molecular recognition elements.

One invention has the advantages of low-cost, minimally invasive approach with high sensitivity and specificity for detecting bone metastasis status - especially one that can be conducted rapidly and conveniently in a physician's office - to enable early detection of cancer stages, a critical unmet need in the challenge to eradicate deaths due to cancer. Additional embodiments include the use of the same device to detect alternate biomarkers associated with response to therapy, which reduces patient exposure to ineffective therapies, minimize the development of ntion includes monitoring disease relapse following successful treatment in a sensitive and rapid way at the 'point-of-care', effectively addressing a critical concern shared by all cancer survivors [9].

In embodiments of the present invention, in the presence of a specific biomarker, detectable particlemolecular recognition element conjugates rapidly selfassemble into agglomerates that are typically more than one order of magnitude larger than their individual components. The size distribution of the agglomerated colloids depends on, among other things, the relative concentration of quantum dot conjugates and antigen molecules. These agglomerates, mediated by antigen recognition, are, in embodiments of the invention, characterized by measuring their light scattering and fluorescence characteristics in an unmodified flow cytometer. Protein antigens angiopoietin-2 and mouse IgG are two examples that can be detected to sub- picomolar concentrations using this embodiment. This invention provides relatively simple techniques to the potential simultaneous detection of multiple antigenic biomarkers directly from physiological media and could be used for early detection and frequent screening of cancers and other diseases.
Luminescence Characterization of Quantum Dots Conjugated with Biomarkers for Early Cancer Detection

To date, detection of the tumor marker CA 125 secreted from ovarian epithelium is the only biomarker available for screening. Detection of CA 125 antigen is based on ELISA and RIA assays used in serologic screening for ovarian cancer and monitoring patient therapeutic responses. However, the sensitivity of the current methods is approximately $70 \%$, thereby greatly limiting its value in mass screening for the disease.

Luminescent semiconductor quantum dots (QDs) conjugated with biomolecules to serve as sensitive probes for early detection of the cancer cells, specifically for ovarian cancer and lung cancer, which represents the most lethal malignancies. The present invention includes a luminescent, bio- conjugate for use in the detection of ovarian cancer, comprising a luminescent semi-conductor nanoparticle and at least one biomarker associated with ovarian cancer. The nanoparticles further comprise a core; and a shell forms a colloidal particle. In an illustrative embodiment, the core of the nanoparticle comprises CdSe and the shell comprises ZnS. Preferably, the QDs-probe for biomarker detection is an antibody associated with CA125.

In another embodiment, the present invention includes a luminescent, bio- conjugate for use in the detection of lung cancer, comprising a luminescent semi-conductor nanoparticle and at least one biomarker associated with lung cancer. The biomarker is an antigen associated with at least one molecule chosen from the group consisting of ADAM10, H2BFQ, AASDHPPT, AB026190, DDX10, OPA1, EKI1, ZWINT, hTERT, VEGF, sIL-2, pSAT3, MAGE, MIF, and Osteopontin [10].

\section{Imaging Hybrid Nanoparticles Used for Cancer Cell Detection}

Robust molecular imaging of cells, tumors, metastases, micro-metastases and the like typically comprises two components: a molecular-specific source of signal (typically provided through a contrast agent) and an imaging system to detect this signal. In recent years, great advances have been made in molecular imaging in small animals using MRI and optical technology to assess novel molecular contrast agents. Each of these techniques has certain advantages and constraints. While MRI provides exceptional anatomic information and depth of imaging, it suffers from limited spatial resolution. Optical imaging yields unprecedented spatial resolution (less than 1 micron) and is inexpensive, robust, and portable, but does not provide the penetration depth, field of view, or anatomic detail achievable with MRI. Thus, it is contemplated that a combination of MRI and optical techniques will address the limitations of both methods, providing an unprecedented range of imaging resolution and penetration depth. 
Embodiments of the invention include approaches for molecular specific optical imaging that combines the advantages of molecularly targeted plasmonic nanoparticles and the ability to magnetically actuate a superparamagnetic particle. This combination is achieved through synthesis of hybrid nanoparticles with a superparamagnetic core surrounded by a optically interrogatable layer, e.g., metal or gold layer. The nanoparticles may be conjugated or operatively coupled with a targeting moiety, such as monoclonal antibodies, antibodies, peptides, small molecules, aptamers and the like, for molecular recognition. A variety of cell features and/or components can be utilized to selectively or specifically target a cell, such as cell surface receptors, lipids, oligosaccharides or essentially any feature or component that is identified to be present on a cell of interest and reduced or absent on non-targeted cells. The hybrid nature of these particles provides for optical contrast enhancement. The addition of the gold layer provides for (1) initially strong optical signal that facilitates detection and digital processing; (2) tunable optical resonances; and (3) a convenient surface for conjugation of probe or targeting molecules or moieties. The core provides a magnetically susceptible component, e.g., iron oxide that can be exploited to periodically actuate cells in the field of view and, therefore, allows use of an external magnetic field for modulation of the optical signal. This approach can increase sensitivity of optical imaging by orders of magnitude [11].

\section{Branched Multifunctional Nanoparticle Conjugates}

The polyglycerol nanoparticle comprise imaging agent, and specific targeting agent can serve as an excellent tumor detection sensor. Linking an optical imaging agent, such as a near-infrared dye, to the nanoparticle enables the in-vivo imaging and tracking of the delivery of nanoparticles. The disclosed nanoparticles are therefore useful as imaging agents, and also provide valuable information about the pharmacokinetics and biodistribution of the nanoparticles. The imaging agents assist in the detection of primary and secondary tumors, and promote the understanding of tumor biology under in-vivo conditions.

Existing problems with targeted nanoparticle delivery are addressed by providing a class of self-assembled nanoparticles containing polyglycerol-drug conjugates as multi-functional nano-platforms. The nanoparticles are suitable for use in medical imaging and therapy. The self-assembled nanoparticles have shown excellent tumor targeting capacity though passive targeting and liganddirected active targeting while maintaining low nonspecific RES uptake.

Embodiments of the disclosed compounds accomplish selective tissue targeting by using a covalently bound targeting agent to exploit up-regulated receptors to deliver a therapeutic agent to a cancer cell selectively [12].

\section{Nanoparticle Biochip Substrates for Cancer Detection}

Biochips are available for use in assessing a wide variety of interactions such as one or more moiety (e.g. bio-molecules, catalyst etc.), either on, or in the presence of a surface. However, in almost all instances, the efficiency or use of biochips is limited, at least in part, by the physical and chemical properties of the surface to which the moieties (bio-molecules and other specific molecules of interest) are attached, as well as the effects on the moieties that result from their interaction with the surface.

A novel 'nano-particle biochip substrate' is disclosed. The 'nano-particle biochip substrate' includes a base of at least one surface, with the top most surface patterned with at least one nano-particle and typically a plurality of nano-particles (also referred to herein as clusters). The nano-particles are attached (pinned, embedded, grown on, or otherwise affixed) on or in the substrate surface, such that they are stable on the surface under conditions under which the nano-particle biochip substrate and nanoparticle biochips are used. The nano-particle biochips can be used for the detection and/or quantification of biomarkers for various cancers at early or late stages [13]. In vivo tumor spectroscopic detection with surfaceenhanced raman nanoparticles tages.

The present patent is generally related to surfaceenhanced Raman spectroscopy nanoparticles, which comprise a metallic nanoparticle core, a Raman reporter and a protective layer.

The nanostructure comprises a core metallic, and gold nanoparticle, a Raman reporter molecule disposed on the surface of the core, and an encapsulating protective layer disposed over the core and the reporter molecule, and wherein the encapsulated reporter molecule has a measurable surface-enhanced Raman spectroscopic signature, allowing the nanostructure to contact a targeted biological cell or tissue, exciting the reporter molecule with a source of radiation, and measuring the surface enhanced Raman spectroscopy spectrum of the nanostructure corresponding to the reporter molecule, thereby detecting the presence of the nanostructure in the targeted cell or tissue.

The target cell may be a cancerous cell of an animal or human subject and the target-specific probe may selected from the group consisting of an antibody, a polypeptide, a polynucleotide, a drug molecule, an inhibitor compound, and a combination thereof, and wherein the targeting probe has an affinity for a marker on the surface of a target cell. Under in vivo conditions, nanostructures according to the disclosure can be delivered to tumors by both a passive targeting mechanism and an active targeting mechanism. In the passive mode, macromolecules and nanometer-sized particles are accumulated preferentially at tumor sites through an enhanced permeability and retention (EPR) effect. This effect is believed to arise from two factors: (a) angiogenic 
tumors that produce vascular endothelial growth factors (VEGF) that hyperpermeabilize the tumor-associated neovasculatures and cause the leakage of circulating macromolecules and small particles; and (b) tumors lack an effective lymphatic drainage system, which leads to subsequent macromolecule or nanoparticle accumulation [14].

\section{METHOD FOR ELECTROCATALYTIC PROTEIN DETECTION}

The present invention provides a method of detecting an analyte in a sample with probe-modified electrodes and measuring an electrocatalytic signal generated by a binding of an analyte in the sample to a probe. In particular, the electrocatalytic signal is generated by a binding of the target protein in the sample to the probe, wherein a change of the signal detected relative to a signal of a control sample comprising no target protein is indicative of the presence of the target protein in the sample. As such, the activity of PSA is the target, peptide known to be cleaved by the complex and modified to bind to the wire is used as a probe, a three-dimensional morphology of nanowires draws analytes towards the electrode and therefore increases the efficiency of the method of the present invention [15].

\section{Detection of Protease and Protease Activity Using a Single Nanocrescent SERS Probe}

In certain embodiments the substrate is a nanocrescent surface enhanced Raman scattering (SERS) probe. The surface enhanced Raman scattering (SERS) probe is comprised of a peptide conjugated to a nanocrescent core and shell, wherein the probe features a sequence, that can be specifically cleaved by a protease (e.g., a protease recognition site), linked to a Raman active tag. This indicator enables detection of proteolytic activity in extremely small volumes and amount. In certain embodiments, the use of a highly focused excitation source allows the detection volume to be only about 10 femtoliter. In various embodiments the actual protease molecule number for the nanomolar samples is less than about 40 molecules, and in certain embodiments close to the single molecule level. Compared to other cancer biomarker detection assays, the present bioconjugated nanocrescent allows the detection of nanomolar concentrations of proteolytically active protease molecules in femtoliter volumes, which is crucial especially for cancer screening at a single cancer cell level [16].

\section{NANO-STRUCTURED DEVICE FOR CANCER CELL DETECTION}

This invention demonstrated a nano-structured device which can be used to detect chemical or biological analyte and cancer or other physiological conditions though the signal change of chemical stress or mass increase.
Accordingly, in detecting cancer or other physiological conditions, there are two ways to use the nano-structured device. One comprising carrier substrate for generating a signal which changes in proportion to a mass increase of the nano-structured device, a transducer film disposed over a surface of the carrier substrate, and a dendrimer structure tethered to the transducer film. The signal generated by the carrier substrate indicates the detection of the cancer or other physiological condition. The dendrimer structure includes a plurality of receptors for binding biomarkers specific to the cancer or other physiological condition to the dendrimer structure, wherein the mass increase proportional to the quantity of the biomarkers binding to the dendrimer structure via the receptors, the mass increase causing the carrier substrate to generate the signal indicating the detection of the cancer or other physiological condition. The other comprising a carrier substrate, a transducer film disposed over a surface of the carrier substrate, and a dendrimer structure tethered to the transducer film. The transducer film generates a first signal in response to a mechanical stress applied thereto, the first signal indicating the detection of the cancer or other physiological condition. The dendrimer structure includes a plurality of receptors for binding biomarkers specific to the presence of cancerous cells or other physiological conditions. Upon interaction of the biomarker with the receptors of the dendrimer structure (functionalized dendrimer structure), a mechanical stress is applied to the transducer film, which is proportional to the strain induced into the dendrimer by the biomarkers binding to the receptors, the mechanical stress causing the transducer film to generate the first signal indicating the detection of the cancer or other physiological condition [17].

\section{SUMMARY}

From the above described patents application on nanosensor, it is not difficult to conclude that nanosensor technology highlights its unique characteristics such as high throughout, high sensitivity and specificity in cancer detection. While it still need take more efforts to optimize the technical parameters of the nanosensor systems for clinical applications. More investigations need to be further conducted to normalize the synthesis routes of nanoparticles and nanosensor, at the same time, targeted ligands to modify the nanoparticles with much higher specificity need to be discovered to get better detection sensitivity.

\section{Acknowledgements}

This work was supported by NSFC (30900345),Programs Foundation of Ministry of Education of China (20090101120155), Startup Foundation of Ministry of Education for Returned Scholars (J20101127) and Zhejiang provincial department of education.

\section{References}

1. Buszewski et al., Biomed. Chromatogr., 2007, 21, 553-566 
http://nanobe.org

2. Cao et al., CHt Rev. Analy. Chem., 2007, 37, 3-13

3. HAICK, Hossam, GANG, Peng. WO 2009144725, 2009.

4. MELKER, Richard J, DENNIS, Donn, Michael. WO 2007086986, 2007.

5. Phillips et al., J Chromatogr. B. Biomed. ScI ASS1, 729, 75, 1999.

6. Phillips et al., J Lab CHn. Med, 136, 243, 2000.

7. HAICK, Hossam. WO 2009053981, 2009.8. HAICK Hossam. WO/2009/013754, 2009.

9. SOMAN, Chinmay Prakash, GIORGIO, Todd Donald. WO 2008124525, 2008.

10. Zhukov Tatyana A, Ostapenko Sergei, Sutphen Rebecca, Lancaster Johnathan, Sellers Thomas A, Zhang, Jin Z. US20060003465, 2006.

11. SOKOLOV Konstantin, MILNER Thomas, LARSON Timothy, BANKSON James, AARON Jesse, OH Jungwan, JI Xiaojun,LI Chun. WO/2008/140624, 2008.

12. NIE Shuming,DUAN Hongwei,KUANG Min. WO/2009/123934),
2009

13. Ure, David A. US20080038830, 2008

14.QUIAN XIMEI, ANSARI DOMINIC, NIE SHUMING WO2008122035,2008.

15. Kelley Shana, O, Roberts Marcel A. WO/2007/094805, 2007.

16. ELLMAN JONATHAN A, LEE LUKE P, LIU GANG L, CHEN FANQING FRANK. WO2008018933, 2008.

17. PALENA Patricia D, BARTO Richard R Jr, BORDERS Tammie L, STUART Jeffrey A. (US). WO/2009/086561, 2009.

Copyright:(c) 2011 W. Shao, et al. This is an openaccess article distributed under the terms of the Creative Commons Attribution License, which permits unrestricted use, distribution, and reproduction in any medium, provided the original author and source are credited. 